\title{
Adaptive Control of Dynamically Complex Networks with Saturation Couplings
}

\author{
Guoliang Wang and Tingting Yan \\ School of Information and Control Engineering, Liaoning Shihua University, Fushun, Liaoning 113001, China \\ Correspondence should be addressed to Guoliang Wang; gliangwang@aliyun.com
}

Received 10 August 2016; Accepted 28 September 2016

Academic Editor: Pilar R. Gordoa

Copyright ( 2016 G. Wang and T. Yan. This is an open access article distributed under the Creative Commons Attribution License, which permits unrestricted use, distribution, and reproduction in any medium, provided the original work is properly cited.

\begin{abstract}
This paper considers the control problem of dynamically complex networks with saturation couplings. Two novel control schemes in terms of adaptive control are presented to deal with such saturation couplings. Based on the robust idea, the underlying complex network is firstly transformed into a strongly connected network having time-varying uncertainty. However, the upper bound of uncertainty is unknown. Because of such an unavailable bound, a kind of adaptive controller added to each node is proposed such that the closed-loop auxiliary network is uniformly bounded. In particular, the original system states are asymptotically stable. Moreover, in order to avoid adding the desired controller to every node, another different kind of adaptive controller based on the pinning control idea is proposed. Compared with the former method, it is only applied to a part of nodes and has a good operability. Finally, a numerical example is provided to show the effectiveness of our results.
\end{abstract}

\section{Introduction}

Complex network is composed of a large number of interconnected dynamical units, which is ubiquitous in nature and human society, such as ecosystem network, biological network, food network, social network, and transportation network. It is regarded as a fundamental tool in understanding a variety of dynamic phenomena which are presented in real worlds $[1,2]$. During the past years, a lot of related problems have been investigated for complex networks, for example, stability and synchronization [3-7], the analysis of complex networks with topological structures [8-11], pinning control [12-14], adaptive control [15-17], impulsive control $[18,19]$, and robust analysis [20-22].

On the other hand, it is known that the topological structure of complex network is important and could determine the characteristic of complex network in addition to controlling them effectively. By investigating the existing references about complex networks, it is found that most of them such as [23-27] were mainly focused on the static networks, whose topological structures were fixed. In order to consider an actual case that the topological structure of complex network changes randomly, the synchronization problem of dynamical networks with switching [28-30] or Markovian jump topologies has been reported in [31-34]. It is said that such results are general but the coupling structures are not completely time-varying. The reason is that the related changes of topology belong to a finite set. Particularly, the synchronization problem of complex network with timevarying topological structures was studied in $[35,36]$. In the above results, it is seen that their couplings are totally time-varying and without any restrictions. However, in many practical applications especially in the real networks with a limited communication capacity, it is difficult to allow them varying abruptly in terms of being randomly large. In this case, saturation phenomenon is a common problem existing in most network systems, which could affect the stability in a large part. To the best of authors' knowledge, no related results are available and could be used to study the related problems of complex networks with saturation couplings. All these observations motivate the current study.

Inspired by the above discussions, the control problem of dynamically complex networks with saturation couplings is studied in this paper, where new adaptive controllers are proposed to handle the saturation couplings. The main contributions of this paper are as follows. (1) By applying 
the robust method, an equivalent description of such a complex network is established, which is actually a strongly connected complex network with time-varying uncertainty. However, the upper bound of uncertainty with norm form is unknown. (2) Due to the bound inaccessible, a new kind of adaptive controller is developed and added to every node, where the estimation of such an unknown bound is given by the given updated law. It is achieved that not only is the closed-loop auxiliary network bounded but also the system states are asymptotically stable. (3) Because of the above adaptive controller related to each node and in order to remove the constraint, another different kind of adaptive pinning controller motivated by the pinning viewpoint is proposed. Compared with the former adaptive controller, it could effectively reduce the number of controllers and is more suitable in some practical applications.

Notation 1. $\mathbb{R}^{n}$ denotes the $n$ dimensional Euclidean space; $\operatorname{diag}\{\cdots\}$ denotes a block-diagonal matrix. $I_{N}$ represents an identity matrix being of $N$ dimensions. $\lambda_{\max }(\cdot)$ is the maximum eigenvalue of matrix. $\|\cdot\|$ denotes the Frobenius norm of a matrix. $*$ denotes an ellipsis for the term induced by symmetry.

\section{Problem Formulation}

Consider a class of complex networks with saturation coupling and described as

$$
\begin{aligned}
\dot{x}_{i}(t)= & f\left(x_{i}(t)\right)+c \sum_{j=1, j \neq i}^{N} \sigma\left(a_{i j}(t)\right)\left(x_{j}(t)-x_{i}(t)\right) \\
& +u_{i}(t), \quad i=1,2, \ldots, N
\end{aligned}
$$

with $x_{i}(t)=\left[x_{i 1}(t), x_{i 2}(t), \ldots, x_{i n}(t)\right]^{T} \in \mathbb{R}^{n}$ representing the state vector of node $i$. Nonlinear function $f: \mathbb{R}^{n} \rightarrow \mathbb{R}^{n}$ is a vector function and describes the dynamics of each node. Positive scalar $c$ is the coupling strength. $u_{i}(t)$ is the control input of node $i . A(t)=\left[a_{i j}(t)\right]_{N \times N}$ is the coupling matrix. Its element is defined as follows: if there is a connection between nodes $i$ and $j$ at time $t,(i \neq j), a_{i j}(t)>0$; otherwise, $a_{i j}(t)=0$. $\sigma\left(a_{i j}(t)\right), i \neq j$, stands for coupling saturation and is defined as follows:

$$
\begin{aligned}
& \sigma\left(a_{i j}(t)\right)= \begin{cases}a_{i j}(t), & \text { if } a_{i j}(t) \in[0, \zeta) \\
\zeta, & \text { otherwise }\end{cases} \\
& \sigma\left(a_{i i}(t)\right)=-\sum_{j=1, j \neq i}^{N} \sigma\left(a_{i j}(t)\right) .
\end{aligned}
$$

In order to make the notation simple, the corresponding coupling matrix is denoted as $\sigma(A(t))=\left[\sigma\left(a_{i j}(t)\right)\right]_{N \times N}$. Without loss of generality, parameter $\zeta$ is assumed to be $\zeta=1$ in this paper. Different from the traditionally complex network whose time-varying coupling is without any restrictions and described by

$$
\begin{aligned}
\dot{x}_{i}(t)= & f\left(x_{\mathrm{i}}(t)\right)+c \sum_{j=1, j \neq i}^{N} a_{i j}(t)\left(x_{j}(t)-x_{i}(t)\right) \\
& +u_{i}(t), \quad i=1,2, \ldots, N
\end{aligned}
$$

the introduction of saturation coupling in complex network (1) comes from the actual network having limited communication capacity. In particular, if $\zeta \rightarrow \infty$, complex network (1) with saturation coupling will become complex network (4) without any constraints on coupling. In other words, it is more actual that it cannot suffer the coupling between nodes arbitrarily large. Instead, there are some restrictions. In this sense, it is said that system (1) is more general and reasonable. Because of the coupling having saturation and nonlinear, the corresponding analysis and synthesis will be complicated. In this paper, by considering the property of saturation coupling (2) with constraint (3) fully, we firstly apply the robust idea to deal with the saturation coupling. Then, complex network (1) is equivalent to

$$
\dot{x}_{i}(t)=f\left(x_{i}(t)\right)+c \sum_{j=1}^{N}\left(\bar{a}_{i j}+\Delta a_{i j}(t)\right) x_{j}(t)+u_{i}(t),
$$

$$
i=1,2 \ldots, N
$$

which can be expressed by

$$
\dot{x}(t)=f(x(t))+c(\bar{A}+\Delta A(t)) \otimes I_{n} x(t)+u(t),
$$

where

$$
\begin{aligned}
& x(t)=\left[x_{1}^{T}(t), x_{2}^{T}(t), \ldots, x_{N}^{T}(t)\right]^{T}, \\
& f(x(t)) \\
& \quad=\left[f^{T}\left(x_{1}(t)\right), f^{T}\left(x_{2}(t)\right), \ldots, f^{T}\left(x_{N}(t)\right)\right]^{T}, \\
& \bar{A}=\left[\begin{array}{crrr}
-\frac{N-1}{2} & \frac{1}{2} & \cdots & \frac{1}{2} \\
* & -\frac{N-1}{2} & \cdots & \frac{1}{2} \\
\vdots & \vdots & \ddots & \vdots \\
* & * & * & -\frac{N-1}{2}
\end{array}\right] .
\end{aligned}
$$

Particularly, $\triangle A(t)$ is time-varying uncertainty and defined as $\Delta A(t)=\left[\Delta a_{i j}(t)\right]_{N \times N}$. Moreover, its element is satisfied:

$$
\begin{aligned}
& \Delta a_{i j}(t) \in\left[-\frac{1}{2}, \frac{1}{2}\right], \quad \forall i \neq j, \\
& \Delta a_{i i}(t)=-\sum_{j=1, j \neq i}^{N} \Delta a_{i j}(t) .
\end{aligned}
$$

Though, its uncertainty could be expressed by (8), it is unknown and inaccessible. Thus, its norm is also unknown and described as

$$
\|\Delta A(t)\| \leq \delta^{*}
$$

where $\delta^{*}>0$ is unavailable. 
Remark 1. It is worth mentioning that, based on the robust method, the originally complex network (1) with saturation couplings (2) and (3) is transformed into an equivalent complex network (5). It is found that the equivalent description is a strongly connected complex network having a time-varying uncertainty. But, the uncertainty is unknown, whose bound is also inaccessible. Then, the difficulty coming from the saturation coupling is avoided. Unfortunately, it is transformed into another difficulty which should be handled. That is how to deal with a complex network with unknown coupling uncertainties. However, it is said that this equivalent transformation would make the control problem of generally complex network (1) considered possibly.

Before giving our main results, some necessary statements are needed here.

Lemma 2 (see [37]). Kronecker product has the following properties:

(i) $(a A) \otimes B=A \otimes(a B)$.

(ii) $A \otimes(B \pm C)=A \otimes B \pm A \otimes C$.

(iii) $(A \otimes B)(C \otimes D)=(A C) \otimes(B D)$.

(iv) $(A \otimes B)^{T}=A^{T} \otimes B^{T}$.

Assumption 3. Assuming that there is a matrix $P=$ $\operatorname{diag}\left\{p_{1}, p_{2}, \ldots, p_{n}\right\}$ with $P>0$ such that

$$
x_{i}^{T}(t) \operatorname{Pf}\left(x_{i}(t)\right) \leq \mu x_{i}^{T}(t) x_{i}(t),
$$

where $\mu>0, x_{i}(t) \in \mathbb{R}^{n}$, and $t \geq 0$.

Remark 4. For this assumption, it is said that it is without loss of generality and could be gotten from reference [25] directly. It has been verified that many chaotic systems, such as Chen system, Lorenz system, and Lü system, satisfy this assumption. Moreover, based on this reference, it is known that matrix $P$ and scalar $\mu$ are given in advance, which should satisfy the above condition.

Definition 5. The complex network (1) with saturation coupling described by (2) and (3) is asymptotically stable, if $\lim _{t \rightarrow \infty}\|x(t)\|^{2}=0$. It is equivalent that the complex network (6) with conditions (8) and (9) is asymptotically stable.

\section{Main Results}

In this section, a kind of adaptive controller guaranteeing the states of the closed-loop complex network asymptotically stable is proposed as

$$
\begin{aligned}
u(t)= & -c\left(K \otimes I_{n}\right) x(t) \\
& -\frac{c^{2} \widehat{\delta}^{2}(t) x(t) x^{T}(t)\left(I_{N} \otimes P\right) x(t)}{c \widehat{\delta}(t) x^{T}(t)\left(I_{N} \otimes P\right) x(t)+\rho(t)},
\end{aligned}
$$

where $K=\operatorname{diag}\left\{k_{1}, k_{2}, \ldots, k_{N}\right\}$ is to be determined and $\rho(t) \epsilon$ $\mathbb{R}^{+}$is any positive uniform continuous bounded function and satisfies

$$
\lim _{t \rightarrow \infty} \int_{t_{0}}^{t} \rho(s) d s \leq \bar{\rho}
$$

with a limited positive constant $\bar{\rho}$. Function $\widehat{\delta}(t) \in \mathbb{R}^{+}$is the estimation of $\delta^{*}$, which is updated by

$$
\dot{\vec{\delta}}(t)=-\eta \rho(t) \widehat{\delta}(t)+c \eta x^{T}(t)\left(I_{N} \otimes P\right) x(t),
$$

where $\eta$ and $\widehat{\delta}_{0}$ are positive constants. Letting $\widetilde{\delta}(t)=\widehat{\delta}(t)-\delta^{*}$, one has

$$
\begin{aligned}
\dot{\tilde{\delta}}(t)= & -\eta \rho(t) \tilde{\delta}(t)+c \eta x^{T}(t)\left(I_{N} \otimes P\right) x(t) \\
& -\eta \rho(t) \delta^{*},
\end{aligned}
$$

where initial condition $\widetilde{\delta}_{0}$ is finite.

Theorem 6. Supposing that Assumption 3 holds, solution $(x, \widetilde{\delta})\left(t, x_{0}, \widetilde{\delta}_{0}, t_{0}\right)$ to the adaptive closed-loop auxiliary system described by (6), (11), and (14) is uniformly bounded, if there exist $k_{i}>0,(i=1,2, \ldots, N)$, such that

$$
\varphi+c \lambda_{\max }(\widetilde{A})<0
$$

where $\varphi=\mu / \min _{1 \leq i \leq n}\left(p_{i}\right)$ and $\widetilde{A}=\bar{A}-K$. In particular, the original system state $x(t)$ is asymptotically stable.

Proof. For the adaptive closed-loop auxiliary system described by (6) with (11) and (14), choose the following Lyapunov function:

$$
V((x, \widetilde{\delta})(t))=\frac{1}{2} x^{T}(t)\left(I_{N} \otimes P\right) x(t)+\frac{1}{2 \eta} \widetilde{\delta}^{2}(t) .
$$

Then, one gets

$$
\begin{aligned}
& \dot{V}((x, \widetilde{\delta})(t))=x^{T}(t)\left(I_{N} \otimes P\right)[f(x(t)) \\
& \left.\quad+c(\bar{A}+\Delta A(t)) \otimes I_{n} x(t)+u(t)\right]+\frac{1}{\eta} \widetilde{\delta}(t) \dot{\tilde{\delta}}(t) \\
& \quad=x^{T}(t)\left(I_{N} \otimes P\right) f(x(t))+c x^{T}(t)\left(I_{N} \otimes P\right)(\bar{A} \\
& \left.\quad \otimes I_{n}\right) x(t)+c x^{T}(t)\left(I_{N} \otimes P\right) \\
& \quad \times\left(\frac{(\Delta A(t))^{T}+\Delta A(t)}{2} \otimes I_{n}\right) x(t)+x^{T}(t)\left(I_{N}\right. \\
& \quad \otimes P) u(t)+\frac{1}{\eta} \widetilde{\delta}(t) \dot{\tilde{\delta}}(t) \leq \mu x^{T}(t) x(t)+c x^{T}(t)(\bar{A}
\end{aligned}
$$




$$
\begin{aligned}
& \otimes P) x(t)+c x^{T}(t)\left(I_{N} \otimes P\right) \\
& \times\left(\frac{(\Delta A(t))^{T}+\Delta A(t)}{2} \otimes I_{n}\right) x(t)+x^{T}(t)\left(I_{N}\right. \\
& \otimes P) u(t)+\frac{1}{\eta} \widetilde{\delta}(t) \dot{\tilde{\delta}}(t) \leq \mu x^{T}(t) x(t)+c x^{T}(t)(\bar{A} \\
& \otimes P) x(t)+c \delta^{*} x^{T}(t)\left(I_{N} \otimes P\right) x(t)+x^{T}(t)\left(I_{N}\right. \\
& \otimes P) u(t)+\frac{1}{\eta} \widetilde{\delta}(t) \dot{\tilde{\delta}}(t)=\mu x^{T}(t) x(t)+c x^{T}(t)(\bar{A} \\
& \otimes P) x(t)+c(\widehat{\delta}(t)-\widetilde{\delta}(t)) x^{T}(t)\left(I_{N} \otimes P\right) x(t) \\
& +x^{T}(t)\left(I_{N} \otimes P\right) u(t)+\frac{1}{\eta} \widetilde{\delta}(t)(-\eta \rho(t) \widetilde{\delta}(t) \\
& \left.+c \eta x^{T}(t)\left(I_{N} \otimes P\right) x(t)-\eta \rho(t) \delta^{*}\right)=\mu x^{T}(t) \\
& \cdot x(t)+c x^{T}(t)(\bar{A} \otimes P) x(t)+c \widehat{\delta}(t) x^{T}(t)\left(I_{N}\right. \\
& \otimes P) x(t)+x^{T}(t)\left(I_{N} \otimes P\right) u(t)+\widetilde{\delta}(t)(-\rho(t) \widetilde{\delta}(t) \\
& \left.-\rho(t) \delta^{*}\right) .
\end{aligned}
$$

Substituting (11) into (17) and further based on the inequality

$$
0<\frac{a b}{a+b} \leq b, \quad \forall a>0, \forall b>0
$$

one has

$$
\begin{aligned}
\dot{V} & ((x, \widetilde{\delta})(t)) \leq \mu x^{T}(t) x(t)+c x^{T}(t)(\bar{A} \otimes P) x(t) \\
& +c \widehat{\delta}(t) x^{T}(t)\left(I_{N} \otimes P\right) x(t)-c x^{T}(t)(K \otimes P) x(t) \\
& -\frac{c^{2} \widehat{\delta}^{2}(t) x^{T}(t)\left(I_{N} \otimes P\right) x(t) x^{T}(t)\left(I_{N} \otimes P\right) x(t)}{c \widehat{\delta}(t) x^{T}(t)\left(I_{N} \otimes P\right) x(t)+\rho(t)} \\
& +\widetilde{\delta}(t)\left(-\rho(t) \widetilde{\delta}(t)-\rho(t) \delta^{*}\right)=\mu x^{T}(t) x(t) \\
& +c x^{T}(t)(\widetilde{A} \otimes P) x(t) \\
& +\frac{c \widehat{\delta}(t) x^{T}(t)\left(I_{N} \otimes P\right) x(t) \rho(t)}{c \widehat{\delta}(t) x^{T}(t)\left(I_{N} \otimes P\right) x(t)+\rho(t)} \\
& +\rho(t)\left(-\widetilde{\delta}^{2}(t)-\delta^{*} \widetilde{\delta}(t)\right) \leq \mu x^{T}(t) x(t) \\
& +c x^{T}(t)(\widetilde{A} \otimes P) x(t)+\rho(t) \\
& +\rho(t)\left(-\widetilde{\delta}^{2}(t)-\delta^{*} \widetilde{\delta}(t)\right) \\
& \leq \lambda_{\max }\left(\mu I_{N n}+c(\widetilde{A} \otimes P)\right)\|x(t)\|^{2}+\rho(t) \varepsilon \\
& \leq\left(\mu+c \lambda_{\max }(\widetilde{A}) \min _{1 \leq i \leq n}\left(p_{i}\right)\right)\|x(t)\|^{2}+\rho(t) \varepsilon
\end{aligned}
$$

where $\varepsilon=1+(1 / 4)\left|\delta^{*}\right|^{2}$. By letting $\breve{x}(t)=\left[x^{T}(t) \widetilde{\delta}^{T}(t)\right]^{T}, \breve{P}=$ $\operatorname{diag}\left\{I_{N} \otimes P, 1 / \eta\right\}$ and based on condition (15), it is obtained that

$$
\dot{V}(\breve{x}(t)) \leq-\gamma\|x(t)\|^{2}+\rho(t) \varepsilon,
$$

where $\gamma=-\left(\mu+c \lambda_{\max }(\widetilde{A}) \min _{1 \leq i \leq n}\left(p_{i}\right)\right)$. Under the above representations, Lyapunov function (16) is equivalent to

$$
V(\breve{x}(t))=\frac{1}{2} \breve{x}^{T}(t) \breve{P} \breve{x}(t) .
$$

Then, it is obvious that

$$
\alpha_{1}(\|\breve{x}(t)\|) \leq V(\breve{x}(t)) \leq \alpha_{2}(\|\breve{x}(t)\|),
$$

where $\alpha_{1}(\|\breve{x}(t)\|)=(1 / 2) \lambda_{\text {min }}(\breve{P})\|x(t)\|^{2}$ and $\alpha_{2}(\|\breve{x}(t)\|)=$ $(1 / 2) \lambda_{\max }(\breve{P})\|x(t)\|^{2}$. Now, we will prove that system state $x(t)$ asymptotically converges to zero. Based on (21), it is got that

$$
V(\breve{x}(t))=V\left(\breve{x}\left(t_{0}\right)\right)+\int_{t_{0}}^{t} \dot{V}(\breve{x}(s)) d s .
$$

Followed from (20), (22), and (23), it is obtained that

$$
\begin{aligned}
0 & \leq \alpha_{1}(\|\breve{x}(t)\|) \\
& \leq \alpha_{2}\left(\left\|\breve{x}\left(t_{0}\right)\right\|\right)-\int_{t_{0}}^{t} \alpha_{3}(\|x(s)\|) d s+\int_{t_{0}}^{t} \varepsilon \rho(s) d s
\end{aligned}
$$

with $\alpha_{3}(\|x(t)\|)=\gamma\|x(t)\|^{2}$. By taking $t \rightarrow \infty$, one has

$$
0 \leq \alpha_{2}\left(\left\|\breve{x}\left(t_{0}\right)\right\|\right)-\lim _{t \rightarrow \infty} \int_{t_{0}}^{t} \alpha_{3}(\|x(s)\|) d s
$$

$$
+\lim _{t \rightarrow \infty} \int_{t_{0}}^{t} \varepsilon \rho(s) d s .
$$

By considering the definition of $\rho(t)$, one concludes

$$
\lim _{t \rightarrow \infty} \int_{t_{0}}^{t} \alpha_{3}(\|x(s)\|) d s \leq \alpha_{2}\left(\left\|\breve{x}\left(t_{0}\right)\right\|\right)+\varepsilon \bar{\rho} .
$$

From (24), it is known that

$$
0 \leq \alpha_{1}(\|\breve{x}(t)\|) \leq \alpha_{2}\left(\left\|\breve{x}\left(t_{0}\right)\right\|\right)+\int_{t_{0}}^{t} \varepsilon \rho(s) d s .
$$

For any $t \geq t_{0}$, one concludes

$$
\sup _{t \in\left[t_{0}, \infty\right)} \int_{t_{0}}^{t} \varepsilon \rho(s) d s \leq \varepsilon \bar{\rho} .
$$

Then, it is obtained that

$$
0 \leq \alpha_{1}(\|\breve{x}(t)\|) \leq \alpha_{2}\left(\left\|\breve{x}\left(t_{0}\right)\right\|\right)+\varepsilon \bar{\rho} .
$$

It means that $\breve{x}(t)$ is uniformly bounded. On the other hand, because $\breve{x}(t)$ is continuous, it is obtained that $\breve{x}(t)$ is uniformly continuous. Then, $x(t)$ is uniformly continuous. From the expression of $\alpha_{3}(\|x(t)\|)$, it is claimed that $\alpha_{3}(\|x(t)\|)$ is uniformly continuous. By applying the Barbalat lemma, one has $\lim _{t \rightarrow \infty} \alpha_{3}(\|x(t)\|)=0$ implying $\lim _{t \rightarrow \infty}\|x(t)\|^{2}=0$. This completes the proof. 
Remark 7. Based on this theorem, it is seen that the adaptive controller (11) plays an important role in achieving the asymptotical stability. Thus, how to obtain the corresponding parameters is an essential problem to be solved. Firstly, for any given function $f\left(x_{i}(t)\right)$, it is necessary to find appropriate parameters $P$ and $\mu$ satisfying Assumption 3. As for the computation of parameter $P$, it could be obtained by selecting some positive scalars $p_{i}, i=1,2, \ldots, n$, while the choice of $\mu$ should satisfy this assumption too. Secondly, coupling strength $c$ and function $\rho(t)$ could be obtained directly and easily, while function $\widehat{\delta}(t)$ is given by (13). Thirdly, based on the condition (15), we could find suitable parameter $k_{i}>0$, $(i=1,2, \ldots, N)$. On the other hand, though the solution to closed-loop system (6) by applying the proposed adaptive controller (11) could achieve zero, it should be added to all the nodes. This will have the application of the proposed method (11) limited. In particular, the node number of complex network is very large. Thus, it is necessary to propose another method to deal with the saturation couplings, where the desired controller could be applied to a part of nodes.

In order to remove the above constraint, another kind of adaptive controller based on the pinning control idea is proposed as

$$
\begin{aligned}
& u_{i}(t)=-c k_{i} x_{i}(t)+v_{i}(t), \quad i=1,2, \ldots, l, \\
& u_{i}(t)=0, \quad i=l+1, l+2, \ldots, N,
\end{aligned}
$$

where

$$
\begin{aligned}
& v_{i}(t) \\
& = \begin{cases}0, & \text { if } \sum_{i=1}^{l} x_{i}^{T}(t) P x_{i}(t)=0, \\
-\frac{c \widehat{\delta}(t) x_{i}(t) \sum_{i=1}^{N} x_{i}^{T}(t) P x_{i}(t)}{\sum_{i=1}^{l} x_{i}^{T}(t) P x_{i}(t)}, & \text { otherwise. }\end{cases}
\end{aligned}
$$

The corresponding updated law is given by

$$
\dot{\widehat{\delta}}(t)=c \alpha \sum_{i=1}^{N} x_{i}^{T}(t) P x_{i}(t)
$$

where $\alpha>0$ is a given positive scalar and $\widehat{\delta}_{0} \in \mathbb{R}^{+}$is the initial condition. Then, we have the following theorem, where fewer nodes are needed to be controlled directly.

Theorem 8. Supposing that Assumption 3 holds, solution $(x, \widetilde{\delta})\left(t, x_{0}, \widetilde{\delta}_{0}, t_{0}\right)$ to the adaptive closed-loop auxiliary system described by (5), (30), and (32) is uniformly bounded, if there exist $k_{i}>0,(i=1,2, \ldots, l)$, such that

$$
\lambda_{\max }\left(\varphi I_{N}+c \breve{A}\right)<0,
$$

where $\breve{A}=\bar{A}-\bar{K}$ and $\bar{K}=\operatorname{diag}\{\underbrace{k_{1}, k_{2}, \ldots, k_{l}}_{l}, 0, \ldots, 0\}$ and $\varphi$ is defined in (15).
Proof. For the adaptive closed-loop auxiliary system described by (5), (30), and (32), choose the following Lyapunov function:

$$
V(t)=\frac{1}{2} \sum_{i=1}^{N} x_{i}^{T}(t) P x_{i}(t)+\frac{1}{2 \alpha} \widetilde{\delta}^{2}(t) .
$$

Then, one has

$$
\begin{aligned}
& \dot{V}((x, \widetilde{\delta})(t))=\sum_{i=1}^{N} x_{i}^{T}(t) P\left[f\left(x_{i}(t)\right)\right. \\
& \left.\quad+c \sum_{j=1}^{N}\left(\bar{a}_{i j}+\Delta a_{i j}(t)\right) x_{j}(t)+u_{i}(t)\right]+\frac{1}{\alpha} \widetilde{\delta}(t) \dot{\tilde{\delta}}(t) \\
& \quad \leq \mu \sum_{i=1}^{N} x_{i}^{T}(t) x_{i}(t)+c \sum_{i=1}^{N} x_{i}^{T}(t) P \sum_{j=1}^{N}\left(\bar{a}_{i j}+\Delta a_{i j}(t)\right) \\
& \cdot x_{j}(t)+\sum_{i=1}^{N} x_{i}^{T}(t) P u_{i}(t)+\frac{1}{\alpha} \widetilde{\delta}(t) \dot{\hat{\delta}}(t) \\
& \quad=\mu \sum_{i=1}^{N} x_{i}^{T}(t) x_{i}(t)+c \sum_{i=1}^{N} x_{i}^{T}(t) P \sum_{j=1}^{N}\left(\bar{a}_{i j}+\Delta a_{i j}(t)\right) \\
& \quad x_{j}(t)-c \sum_{i=1}^{l} k_{i} x_{i}^{T}(t) P x_{i}(t)+\sum_{i=1}^{l} x_{i}^{T}(t) P v_{i}(t)+\frac{1}{\alpha}
\end{aligned}
$$

$$
\begin{aligned}
& \cdot \widetilde{\delta}(t) \dot{\widehat{\delta}}(t) \leq \frac{\mu}{\min _{1 \leq i \leq n}\left(p_{i}\right)} \sum_{i=1}^{N} x_{i}^{T}(t) P x_{i}(t) \\
& +c \sum_{i=1}^{N} x_{i}^{T}(t) P \sum_{j=1}^{N}\left(\bar{a}_{i j}+\Delta a_{i j}(t)\right) x_{j}(t) \\
& -c \sum_{i=1}^{l} k_{i} x_{i}^{T}(t) P x_{i}(t)+\sum_{i=1}^{l} x_{i}^{T}(t) P v_{i}(t)+\frac{1}{\alpha} \widetilde{\delta}(t)
\end{aligned}
$$

$\cdot \dot{\hat{\delta}}(t)$.

Letting $\tilde{x}_{j}(t)=\left(x_{1 j}(t), x_{2 j}(t), \ldots, x_{N j}(t)\right)^{T} \in \mathbb{R}^{N}, j=$ $1,2, \ldots, n$, it is obtained that

$$
\begin{aligned}
& \dot{V}((x, \tilde{\delta})(t)) \\
& \leq \frac{\mu}{\min _{1 \leq i \leq n}\left(p_{i}\right)} \sum_{i=1}^{N} x_{i}^{T}(t) P x_{i}(t) \\
& \quad+c \sum_{j=1}^{n} p_{j} \tilde{x}_{j}^{T}(t) \bar{A} \widetilde{x}_{j}(t) \\
& \quad+c \sum_{j=1}^{n} p_{j} \tilde{x}_{j}^{T}(t) \frac{(\Delta A(t))^{T}+\Delta A(t)}{2} \widetilde{x}_{j}(t) \\
& \quad-c \sum_{j=1}^{n} p_{j} \tilde{x}_{j}^{T}(t) \bar{K} \widetilde{x}_{j}(t)+\sum_{i=1}^{l} x_{i}^{T}(t) P v_{i}(t)
\end{aligned}
$$




$$
\begin{aligned}
& +\frac{1}{\alpha} \widetilde{\delta}(t) \dot{\hat{\delta}}(t) \\
\leq & \varphi \sum_{j=1}^{n} p_{j} \widetilde{x}_{j}^{T}(t) \widetilde{x}_{j}(t)+c \sum_{j=1}^{n} p_{j} \tilde{x}_{j}^{T}(t) \breve{A} \widetilde{x}_{j}(t) \\
& +c \delta^{*} \sum_{j=1}^{n} p_{j} \widetilde{x}_{j}^{T}(t) \tilde{x}_{j}(t)+\sum_{i=1}^{l} x_{i}^{T}(t) P v_{i}(t) \\
& +\frac{1}{\alpha} \widetilde{\delta}^{n}(t) \dot{\hat{\delta}}(t) \\
= & \sum_{j=1}^{n} p_{j} \widetilde{x}_{j}^{T}(t)\left(\varphi I_{N}+c \breve{A}\right) \widetilde{x}_{j}(t) \\
& +c \delta^{*} \sum_{j=1}^{n} p_{j} \widetilde{x}_{j}^{T}(t) \widetilde{x}_{j}(t)-c \widehat{\delta}(t) \sum_{i=1}^{N} x_{i}^{T}(t) P x_{i}(t) \\
& +\frac{1}{\alpha}\left(\widehat{\delta}(t)-\delta^{*}\right) \alpha c \sum_{i=1}^{N} x_{i}^{T}(t) P x_{i}(t) \\
= & \sum_{j=1}^{n} p_{j} \widetilde{x}_{j}^{T}(t)\left(\varphi I_{N}+c \breve{A}\right) \widetilde{x}_{j}(t) \leq 0 .
\end{aligned}
$$

Then, it is obtained that $x(t)$ is uniformly bounded. This completes the proof.

Remark 9. It is worth mentioning that, different from Theorem 6, the proposed adaptive controller (30) is not necessarily added to every node. In other words, the stabilization of complex network (1) could be achieved by only controlling a fraction of nodes. Thus, it is very suitable to some complex networks having very large nodes. Moreover, it is said that the selected nodes and their number $l$ are very close to the network dynamics. In other words, the effects based on different selected nodes and node number $l$ will be distinct, even for same complex network. Without loss of generality, only two kinds of complex networks are mentioned here. Because of the nodes of small-world network connected with same probability, it is obvious that the more nodes are controlled, the better performance will be achieved. However, this phenomenon is not true in scale-free network. It means the better performance cannot be obtained by simply controlling nodes as many as possible. This is mainly determined by the structure of scale-free network. In order to get the better performance, we should select the nodes with higher degrees to control. Thus, which and how many nodes to be selected depend on the complex network closely and should be considered concretely. Finally, it is also to point out that, due to only a few nodes controlled, only the bound stability of the closed-loop system could be guaranteed. This is different from Theorem 6. Based on these demonstrations, it is said that both of them are useful to stabilize the complex network with saturation coupling. However, the detailed applications of them should be considered in the concrete situations.

\section{Numerical Example}

Example 1. Consider a complex network with four nodes, each of which is the Sprott G chaotic system [38] and described as

$$
\begin{aligned}
& \dot{x}_{1}(t)=0.4 x_{1}+x_{3}, \\
& \dot{x}_{2}(t)=x_{1} x_{3}-x_{2}, \\
& \dot{x}_{3}(t)=-x_{1}+x_{2} .
\end{aligned}
$$

The chaotic attractor of the Sprott G system is shown in Figure 1. By using the method [25], it is known that one could select $P=I_{3}$ and $\mu=4.5619$ to satisfy Assumption 3. Here, without loss of generality, the time-varying coupling matrix is given by

$$
A(t)=\left[\begin{array}{cccc}
-\sin t-1.5 & 1+\sin t & 0.5 & 0 \\
0.2 & e^{-t}-\sin t-2.2 & 1-e^{-t} & 1+\sin t \\
4 e^{-0.1 t} & 0 & -4 e^{0.1-t}-\arctan t & \arctan t \\
1 & \tanh t & 0.3 & -\tanh t-1.3
\end{array}\right]
$$

Based on the saturation description (2) with (3), the corresponding $\sigma(A(t))$ is

$$
\begin{aligned}
& \sigma(A(t)) \\
& =\left[\begin{array}{cccc}
\bar{a}_{11}(t) & \sigma(1+\sin t) & 0.5 & 0 \\
0.2 & \bar{a}_{22}(t) & 1-e^{-t} & \sigma(1+\sin t) \\
\sigma\left(4 e^{-0.1 t}\right) & 0 & \bar{a}_{33}(t) & \sigma(\arctan t) \\
1 & \sigma(\tanh t) & 0.3 & \bar{a}_{44}(t)
\end{array}\right],
\end{aligned}
$$

where $\bar{a}_{i i}(t)=\sum_{j=1, j \neq i}^{4} \sigma\left(a_{i j}(t)\right), i=1,2,3,4$. If we select $c=11.6$ and $k_{i}=6$, it is known that condition (15) holds. Under initial condition $x_{0}=\left[\begin{array}{lll}0.1 & -0.5 & 0.3\end{array}\right]^{T}$, and letting $\eta_{0}=0.2$ and $\rho(t)=e^{-4 t}$, one could construct the adaptive controller (11) and updated law (13). After applying the above adaptive controller, the curves of the closed-loop auxiliary system are given in Figure 2. The estimated parameter $\widehat{\delta}(t)$ with $\widehat{\delta}_{0}=0.3$ is depicted in Figure 3 . From these simulations, it is seen that the proposed adaptive controller is useful. 


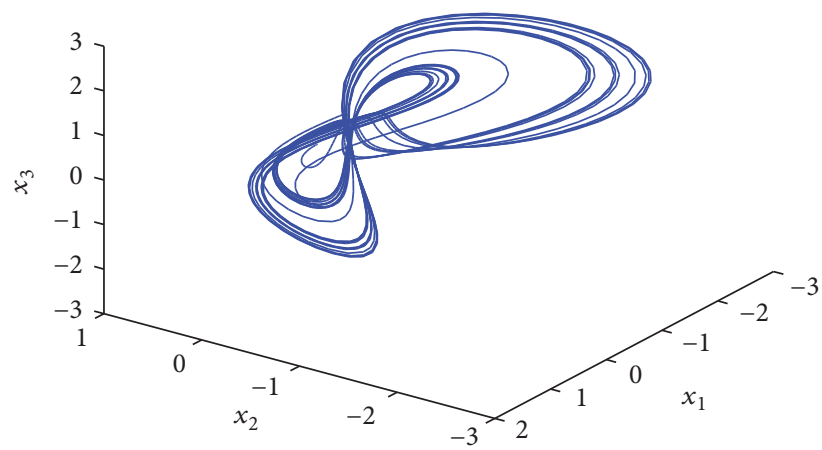

FIGURE 1: The chaotic attractor of the Sprott G system.

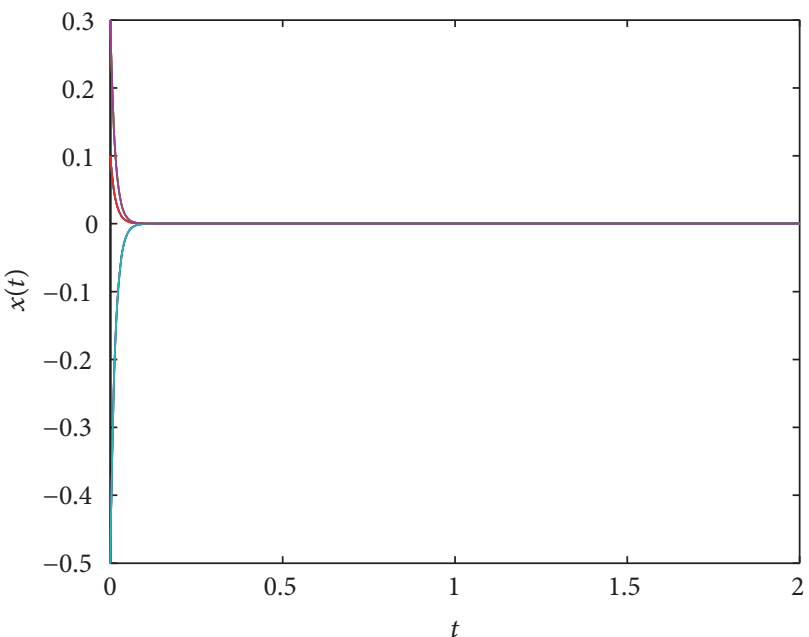

FIGURE 2: The state response of the closed-loop system by controller (11).

Next, we will design an adaptive controller (30) only added to one node to stabilize the above complex network. By selecting $\alpha=0.2$ where the other parameters are the same as the above ones, it is concluded that condition (33) is also satisfied. Then, the corresponding adaptive controller (30) in addition to the updated law (32) could be constructed. After applying the above controller, the simulations of the closed-loop auxiliary system are given in Figures 4 and 5, respectively. Based on the simulations, it is seen that both controllers obtained by Theorems 6 and 8 are useful in terms of stabilizing such a complex network. Moreover, it is obtained that the convergence property of Theorem 6 is better, while fewer nodes are needed in the method of Theorem 8. Thus, it is said that the utility of Theorems 6 and 8 should be considered in the concrete situations.

\section{Conclusions}

In this paper, we have investigated the stabilization problem of complex networks with saturation coupling, whose saturation coupling is handled by the adaptive control method. By exploiting the robust viewpoint, an equivalent description

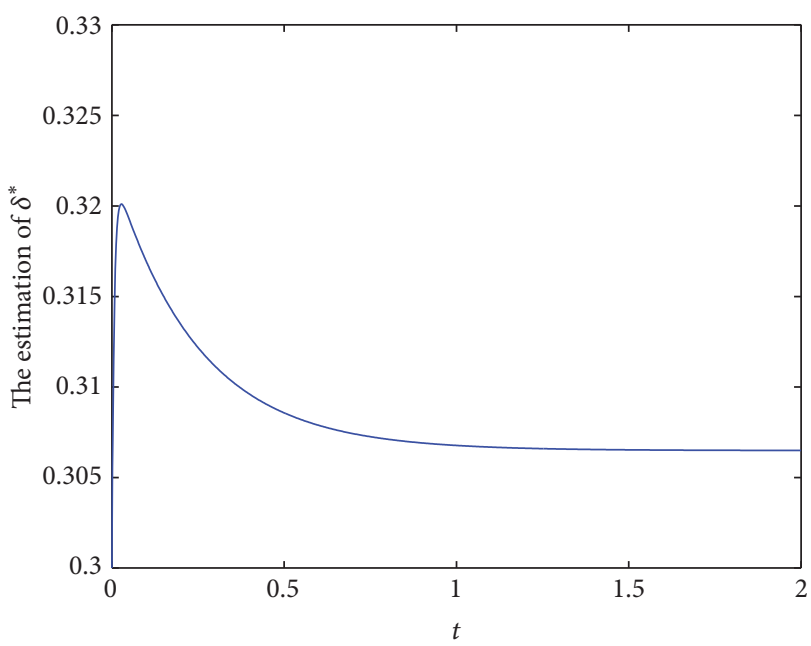

Figure 3: The curve of estimation $\widehat{\delta}(t)$ by updated law (13).

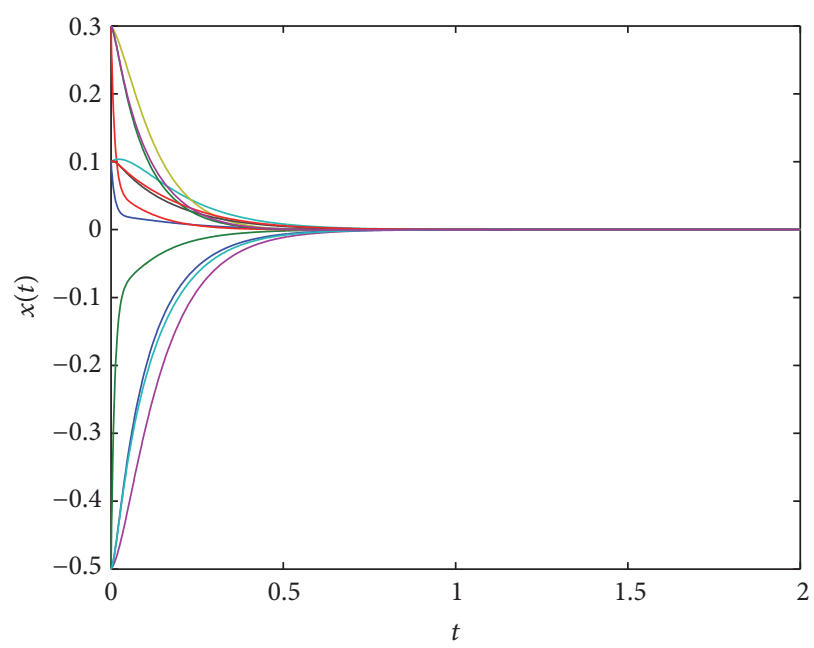

FIGURE 4: The state response of the closed-loop system by controller (30).

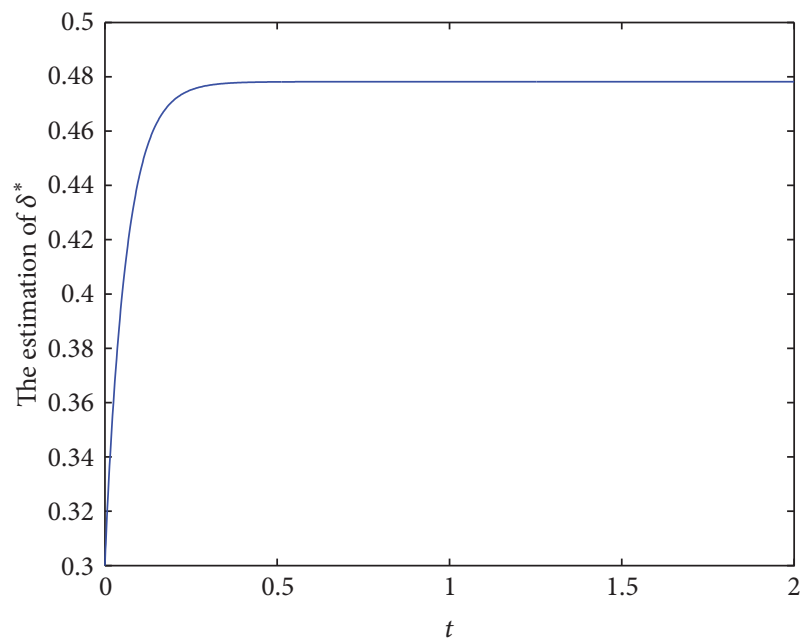

FIgURE 5: The curve of estimation $\widehat{\delta}(t)$ by updated law (32). 
of such a complex network is established, which is transformed to a strongly connected network having time-varying coupling uncertainty. However, the uncertainty is unknown in addition to its bound unavailable. Based on the proposed model, an adaptation law is proposed to estimate the unknown parameter. By applying the updated value, a kind of adaptive controller is proposed that the estimated parameter is bounded, whereas the system state is asymptotically stable. Because of the above adaptive controller added to each node and having some application restrictions, another different adaptive controller which could be added to a fraction of nodes is proposed based on the pinning control idea. Finally, the effectiveness of the theoretical results is illustrated by a numerical example.

\section{Competing Interests}

The authors declare that there is no conflict of interests regarding the publication of this paper.

\section{Acknowledgments}

This work was supported by the National Natural Science Foundation of China under Grants 61104066, 61374043, and 61473140, the China Postdoctoral Science Foundation funded project under Grant 2012M521086, the Program for Liaoning Excellent Talents in University under Grant LJQ2013040, and the Natural Science Foundation of Liaoning Province under Grant 2014020106.

\section{References}

[1] D. J. Watts and S. H. Strogatz, "Collective dynamics of 'smallworld’ networks," Nature, vol. 393, no. 6684, pp. 440-442, 1998.

[2] A.-L. Barabási and R. Albert, "Emergence of scaling in random networks," Science, vol. 286, no. 5439, pp. 509-512, 1999.

[3] T. Liu, J. Zhao, and D. J. Hill, "Exponential synchronization of complex delayed dynamical networks with switching topology," IEEE Transactions on Circuits and Systems I: Regular Papers, vol. 57, no. 11, pp. 2967-2980, 2010.

[4] T. H. Lee, D. H. Ji, J. H. Park, and H. Y. Jung, "Decentralized guaranteed cost dynamic control for synchronization of a complex dynamical network with randomly switching topology," Applied Mathematics and Computation, vol. 219, no. 3, pp. 9961010, 2012.

[5] W. Yu, G. Chen, J. Lu, and J. Kurths, "Synchronization via pinning control on general complex networks," SIAM Journal on Control and Optimization, vol. 51, no. 2, pp. 1395-1416, 2013.

[6] T. Liu, D. J. Hill, and J. Zhao, "Output synchronization of dynamical networks with incrementally-dissipative nodes and switching topology," IEEE Transactions on Circuits and Systems. I. Regular Papers, vol. 62, no. 9, pp. 2312-2323, 2015.

[7] F. Wang, Y. Yang, M. Hu, and X. Xu, "Projective cluster synchronization of fractional-order coupled-delay complex network via adaptive pinning control," Physica A: Statistical Mechanics and Its Applications, vol. 434, pp. 134-143, 2015.

[8] S. Boccaletti, V. Latora, Y. Moreno, M. Chavez, and D.-U. Hwang, "Complex networks: structure and dynamics," Physics Reports, vol. 424, no. 4-5, pp. 175-308, 2006.
[9] M. Rohden, A. Sorge, D. Witthaut, and M. Timme, "Impact of network topology on synchrony of oscillatory power grids," Chaos, vol. 82, no. 1, pp. 279-312, 2014.

[10] X. L. Yang and T. Wei, "Revealing network topology and dynamical parameters in delay-coupled complex network subjected to random noise," Nonlinear Dynamics, vol. 82, no. 1, pp. 319-332, 2015.

[11] H. Shen, J. H. Park, and Z.-G. Wu, "Reliable mixed passive and $H_{\infty}$ filtering for semi-Markov jump systems with randomly occurring uncertainties and sensor failures," International Journal of Robust and Nonlinear Control, vol. 25, no. 17, pp. 32313251, 2015.

[12] M. Ma, J. Cai, and J. Zhou, "Adaptive practical synchronisation of Lagrangian networks with a directed graph via pinning control," IET Control Theory and Applications, vol. 9, no. 14, pp. 2157-2164, 2015.

[13] C. Yu, J. Qin, and H. Gao, "Cluster synchronization in directed networks of partial-state coupled linear systems under pinning control," Automatica, vol. 50, no. 9, pp. 2341-2349, 2014.

[14] Q. Song and J. Cao, "On pinning synchronization of directed and undirected complex dynamical networks," IEEE Transactions on Circuits and Systems I: Regular Papers, vol. 57, no. 3, pp. 672-680, 2010.

[15] P. DeLellis, M. D. Bernardo, and F. Garofalo, "Adaptive pinning control of networks of circuits and systems in lur'e form," IEEE Transactions on Circuits and Systems I: Regular Papers, vol. 60, no. 11, pp. 3033-3042, 2013.

[16] H. Su, Z. Rong, M. Z. Q. Chen, X. Wang, G. Chen, and H. Wang, "Decentralized adaptive pinning control for cluster synchronization of complex dynamical networks," IEEE Transactions on Cybernetics, vol. 43, no. 1, pp. 394-399, 2013.

[17] Z.-Y. Sun and Y.-G. Liu, "Adaptive control design for a class of uncertain high-order nonlinear systems with time delay," Asian Journal of Control, vol. 17, no. 2, pp. 535-543, 2015.

[18] J. Yu, C. Hu, H. Jiang, and Z. Teng, "Stabilization of nonlinear systems with time-varying delays via impulsive control," Neurocomputing, vol. 125, no. 3, pp. 68-71, 2014.

[19] F. Wang, Y. Yang, A. Hu, and X. Xu, "Exponential synchronization of fractional-order complex networks via pinning impulsive control," Nonlinear Dynamics, vol. 82, no. 4, pp. 19791987, 2015.

[20] D. S. Callaway, M. E. J. Newman, S. H. Strogatz, and D. J. Watts, "Network robustness and fragility: percolation on random graphs," Physical Review Letters, vol. 85, no. 25, pp. 5468-5471, 2000.

[21] X. F. Wang and G. Chen, "Synchronization in scale-free dynamical networks: robustness and fragility," IEEE Transactions on Circuits and Systems. I. Fundamental Theory and Applications, vol. 49, no. 1, pp. 54-62, 2002.

[22] C.-L. Pu, W.-J. Pei, and A. Michaelson, "Robustness analysis of network controllability," Physica A: Statistical Mechanics and its Applications, vol. 391, no. 18, pp. 4420-4425, 2012.

[23] Q. Wang, Z. Duan, G. Chen, and Z. Feng, "Synchronization in a class of weighted complex networks with coupling delays," Physica A: Statistical Mechanics and Its Applications, vol. 387, no. 22, pp. 5616-5622, 2008.

[24] H. Pan, X.-H. Nian, and W.-H. Gui, "Synchronization in dynamic networks with time-varying delay coupling based on linear feedback controllers," Acta Automatica Sinica, vol. 36, no. 12, pp. 1766-1772, 2010. 
[25] X. Wu and H. Lu, "Hybrid synchronization of the general delayed and non-delayed complex dynamical networks via pinning control," Neurocomputing, vol. 89, pp. 168-177, 2012.

[26] Q. Ma and J. Lu, "Cluster synchronization for directed complex dynamical networks via pinning control," Neurocomputing, vol. 101, pp. 354-360, 2013.

[27] M. Fang, "Synchronization for complex dynamical networks with time delay and discrete-time information," Applied Mathematics and Computation, vol. 258, pp. 1-11, 2015.

[28] J. Zhao, D. J. Hill, and T. Liu, "Synchronization of complex dynamical networks with switching topology: a switched system point of view," Automatica, vol. 45, no. 11, pp. 2502-2511, 2009.

[29] H.-B. Min, Z.-G. Liu, Y. Liu, S.-C. Wang, and Y.-L. Yang, "Coordination control of networked Euler-Lagrange systems with possible switching topology," Acta Automatica Sinica, vol. 39, no. 7, pp. 1003-1010, 2013.

[30] H.-B. Guo, H.-Y. Li, W.-C. Zhong, S.-J. Zhang, and X.-B. Cao, "Adaptive synchronization of networked Euler-Lagrange systems with directed switching topology," Acta Automatica Sinica, vol. 40, no. 11, pp. 2541-2548, 2014.

[31] X. Yang, J. Cao, and J. Lu, "Synchronization of randomly coupled neural networks with Markovian jumping and timedelay," IEEE Transactions on Circuits and Systems I: Regular Papers, vol. 60, no. 2, pp. 363-376, 2013.

[32] Z.-X. Li, J. H. Park, and Z.-G. Wu, "Synchronization of complex networks with nonhomogeneous Markov jump topology," Nonlinear Dynamics, vol. 74, no. 1-2, pp. 65-75, 2013.

[33] X. Yang and J. Lu, "Finite-time synchronization of coupled networks with Markovian topology and impulsive effects," IEEE Transactions on Automatic Control, vol. 61, no. 8, pp. 2256-2261, 2016.

[34] H. Shen, J. H. Park, Z.-G. Wu, and Z. Zhang, "Finite-time $H_{\infty}$ synchronization for complex networks with semi-Markov jump topology," Communications in Nonlinear Science and Numerical Simulation, vol. 24, no. 1-3, pp. 40-51, 2015.

[35] W. Yu, P. DeLellis, G. Chen, M. Di Bernardo, and J. Kurths, "Distributed adaptive control of synchronization in complex networks," IEEE Transactions on Automatic Control, vol. 57, no. 8, pp. 2153-2158, 2012.

[36] S. Zheng, "Adaptive-impulsive projective synchronization of drive-response delayed complex dynamical networks with time-varying coupling," Nonlinear Dynamics, vol. 67, no. 4, pp. 2621-2630, 2012.

[37] A. N. Langville and W. J. Stewart, "The Kronecker product and stochastic automata networks," Journal of Computational and Applied Mathematics, vol. 167, no. 2, pp. 429-447, 2004.

[38] J. C. Sprott, "Some simple chaotic flows," Physical Review E. Statistical, Nonlinear, and Soft Matter Physics, vol. 50, no. 2, pp. R647-R650, 1994. 


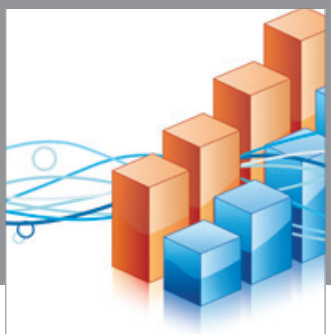

Advances in

Operations Research

vatem alat4

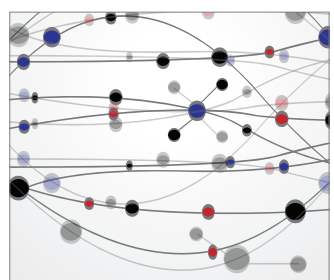

\section{The Scientific} World Journal
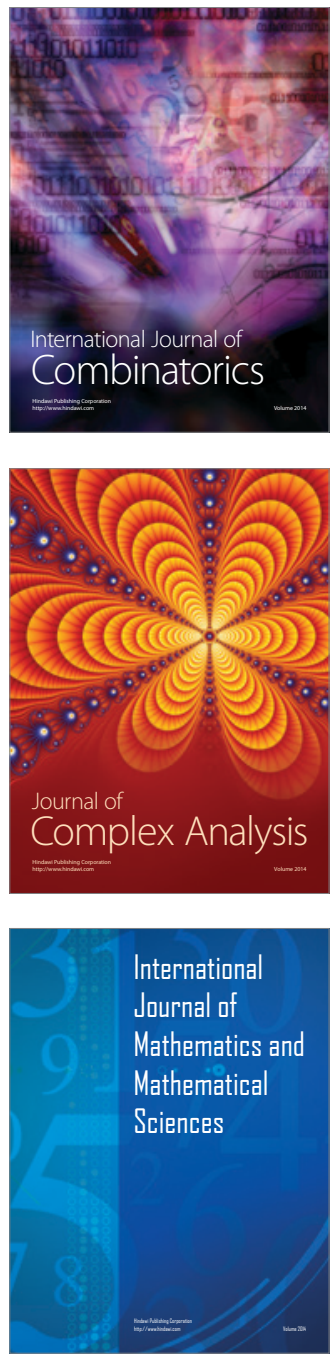
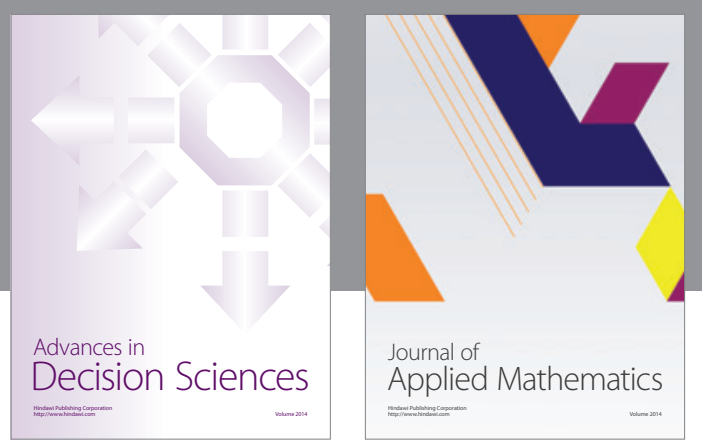

Algebra

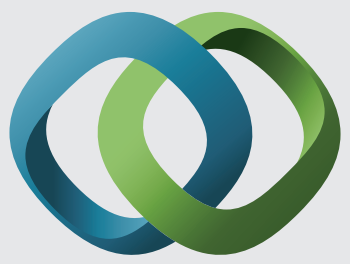

\section{Hindawi}

Submit your manuscripts at

http://www.hindawi.com
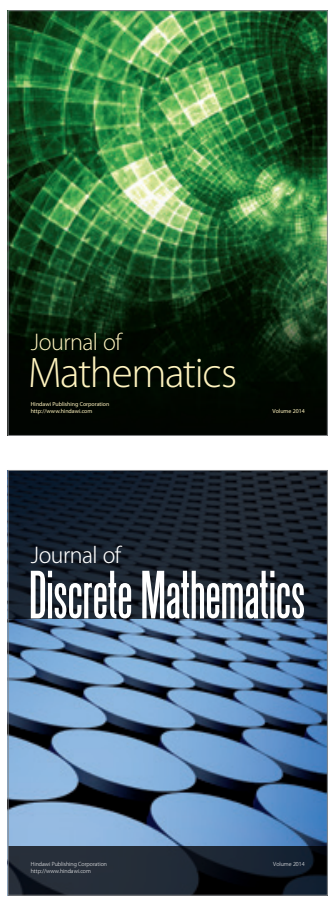

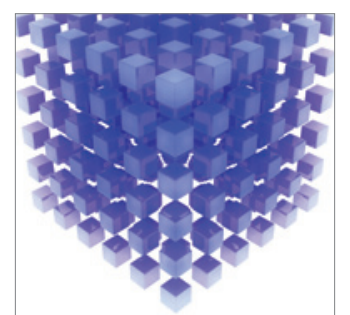

Mathematical Problems in Engineering
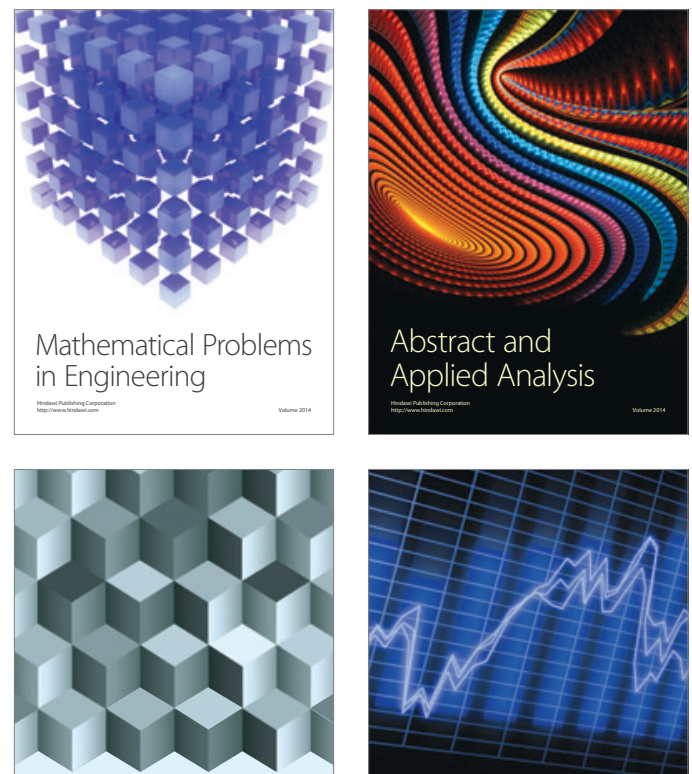

Journal of

Function Spaces

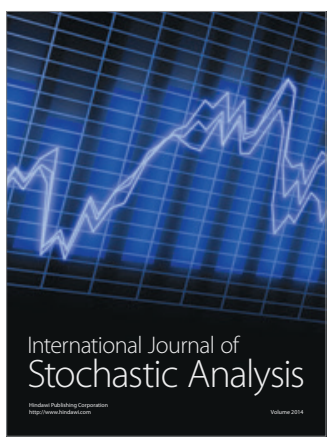

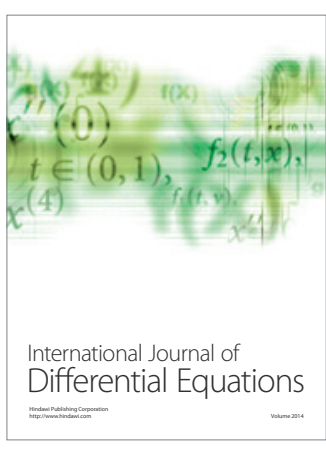
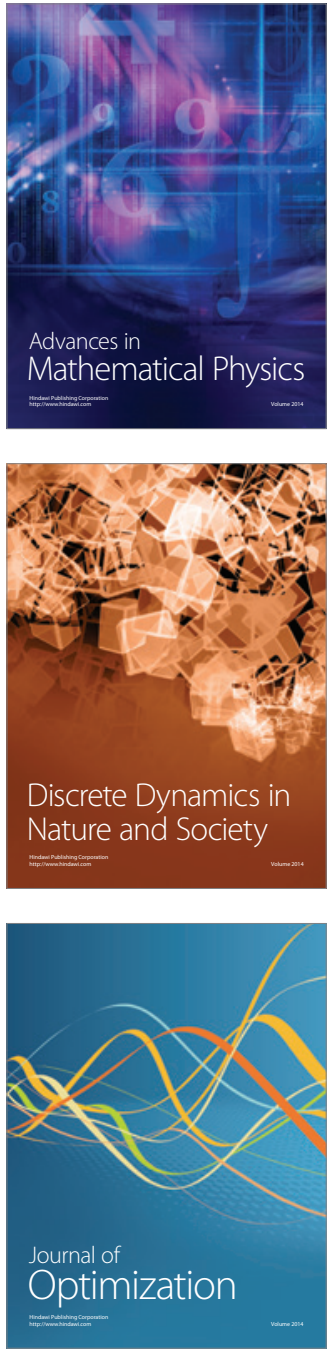